\title{
Complementary approaches with and without a Fourier plane for optical image processing education
}

\author{
A. Márquez, C. Neipp, S. Gallego, M. Ortuño, I. Pascual, \\ et al.
}

A. Márquez, C. Neipp, S. Gallego, M. Ortuño, I. Pascual, M. J. Yzuel, A. Beléndez, J. Campos, "Complementary approaches with and without a Fourier plane for optical image processing education," Proc. SPIE 9664, Ninth International Topical Meeting on Education and Training in Optics and Photonics, 966410 (24 October 2005); doi: 10.1117/12.2207742

SPIE Event: Ninth International Topical Meeting on Education and Training in Optics and Photonics, 2005, Marseille, France 


\title{
Ref ETOP073
}

\section{Complementary approaches with and without a Fourier plane for optical image processing education}

\author{
A. Márquez ${ }^{1 *}$, C. Neipp ${ }^{1}$, S. Gallego ${ }^{1}$, M. Ortuño ${ }^{1}$, I. Pascual ${ }^{2}$, M.J. Yzuel $^{3}$, A. Beléndez $^{1}$, J. \\ Campos $^{3}$ \\ ${ }^{1}$ Depto. de Física, Ingeniería de Sistemas y Teoría de la Señal, Universidad de Alicante, \\ Spain \\ ${ }^{2}$ Departamento Interuniversitario de Óptica, Universidad de Alicante, Spain \\ ${ }^{3}$ Departamento de Física, Universidad Autónoma de Barcelona, Spain \\ *Tel.: +34-96-5903651; Fax: +34-96-5909750; E-mail: amarquez@dfists.ua.es
}

\begin{abstract}
We present two laboratory experiments on optical image processing which show complementary modeling approaches. One experiment is based on an optical correlator, where a physical Fourier plane can be identified, and the other is based on the angular response exhibited by a volume grating (Bragg processing) where no Fourier plane is available. In the correlator the frequency content can be visualized as intensity variations in the Fourier plane, whereas in Bragg processing the angular plane waves decomposition of the object is the intuitive approach. Then we have two different approaches to synthesize the transfer function of the system. The combination of the two experiments in a student's lab helps for a deeper understanding of optical image processing, its linear systems mathematical background, and some aspects of volume holography.
\end{abstract}

\section{Keywords}

Fourier Transform, Image Processing, Bragg processing, Optical Correlator, Linear System, Volume Holography.

\section{Introduction}

\section{Summary}

Optical image processing [1] can be considered an area with an important technological potential, especially when combined with increasingly better optoelectronics devices, such as spatial light modulators or CCD/CMOS cameras. From the educational point of view it is an effective method of introducing the concept of parallelism in computing, inherent in optics. Furthermore it is an efficient and elegant method to present the linear systems theory [2], common to a large number of science and engineering fields, since the lab experiments provide a direct visualization of linearity, superposition, space-shift invariance, direct space domain and frequency (Fourier) domain.

The usual scheme used in optical image processing takes advantage of direct manipulation in the Fourier plane of the frequency content of the object. In the Fourier plane we place a filter, which multiplies the Fourier transform of the object point by point. This would be made in the Vander Lugt type correlators. A variation of this scheme is used in the Joint-transform correlator but we do still visualize a Fourier plane. A different strategy was proposed at the end of the 70s, dedicated to modify the angular plane waves spectrum of the object with no need for a Fourier plane [3]. This image processing strategy, so-called Bragg processing, takes advantage of the characteristics of the angular response in volume holography [4,5]. In the next Section we show two different setups and the possibilities they offer to demonstrate in a students lab optical image processing with and without a Fourier plane. 


\section{Experimental realization of the two approaches}

In Fig. 1(a) we show a modified version of a Vander Lugt converging correlator, as proposed in Ref. [2], with two arms that allow the display of the Fourier plane and the final plane simultaneously. This can be done because we insert a beam-splitter in the light trajectory. By means of the lens L3 we project the Fourier plane onto the screen or onto a CCD camera. Lens L2 projects the Fourier Transform of the Fourier plane onto the CCD camera. In the Fourier plane we put the corresponding frequency filter $H(u)$ multiplying the frequency spectrum $F(u)$ of the object. Thus the frequency content $G(u)$ of the final image is given by $G(u)=F(u) H(u)$. In this type of setups we are able to directly specify in a certain plane the transfer function $H(u)$ of the system.
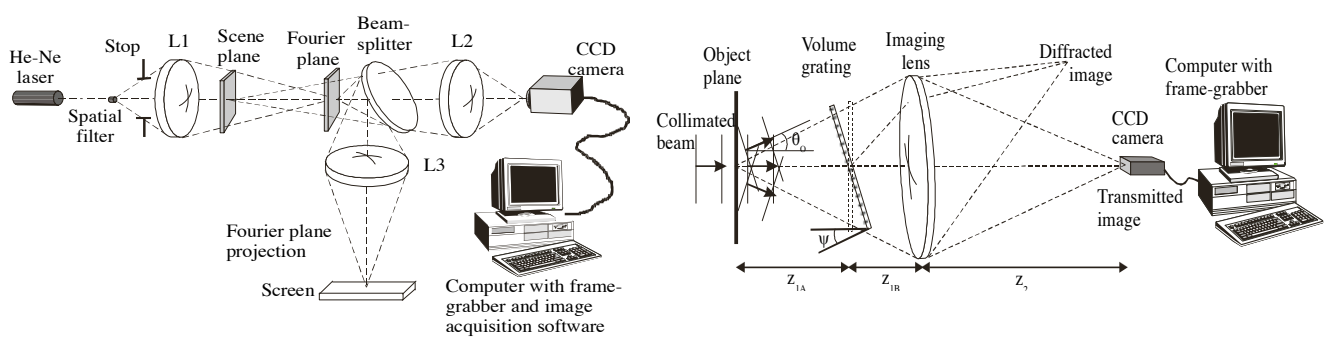

Fig.1. Experimental setups. (a) Double arm convergent correlator. (b) Imaging setup with a volume grating.

In Fig. 1(b) we show a one lens imaging setın where we have introduced a volume grating. (a) volume grating generates a transmitte (b) \& a diffracted order and we obtain two anrerent images at the output plane. Spatial mitering of the object frequency content is accomplished without a physical Fourier plane but through the modification of the angular plane waves spectrum of the object by the volume grating. As given by the Kogelnik's coupled wave theory [4] the diffraction efficiency of a volume grating varies with the angle of incidence. The efficiency as a function of the angle will act as the transfer function of this system.

Let us consider the angles $\theta 0$ and $\psi$, with respect to the optical axis in the optical system (Fig. 1(b)), for the plane waves spectrum of an input object and for the orientation of the grating. With a proper substitution [5] we can rewrite the angular response of the grating as frequency transfer functions for the transmitted $H_{0}(u)$ and the diffracted order $H_{1}(u)$, where $u=\sin \theta_{o} / \lambda_{0}$. Thus, the frequency contents of the transmitted $G_{0}(u)$ and the diffracted images $G_{1}(u)$ are given by: $G_{0}(u)=F(u) H_{0}(u)$ and $G_{1}(u)=F(u) H_{1}(u)$ respectively. We note that these filtering operations are not dependent on the distance between the object and the grating. Therefore we can construct very compact processors where the grating is in close contact with the object. The function of the lens is simply to image the filtered object onto a final plane. An interesting situation arises when the grating exhibits maximum diffraction efficiency and it is oriented at the Bragg angle, $\psi=\theta$ Bragg. Then the DC component of the object is addressed in the direction of the diffracted order and $H_{0}(u)$ and $H_{1}(u)$ correspond respectively to a high-pass and a low-pass filter [5]. 

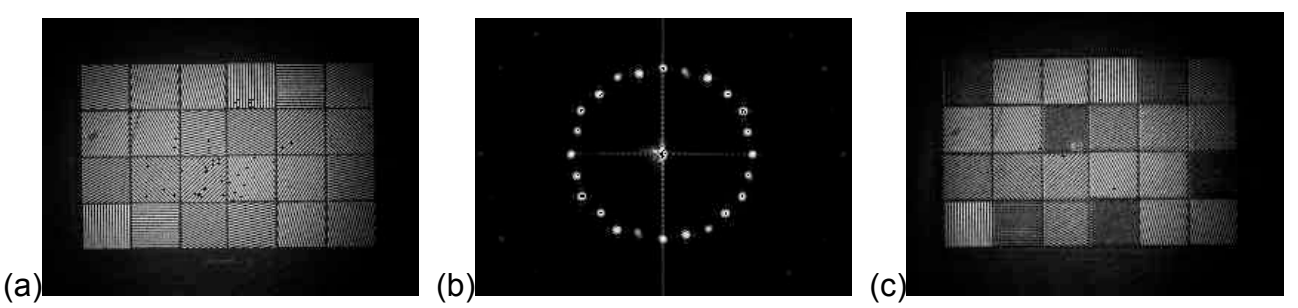

Fig.2. (a) Scene; (b) Diffraction pattern. (c) Reconstructed signal with a filter rejecting the orientations $+90^{\circ},+75^{\circ}$ and $-75^{\circ}$.

Let us show in Fig. 2 and 3 some experimental images obtained respectively with the setups in Fig. 1(a) and 1(b). With the double arm correlator we see in Fig. 2(a) the input object, composed by several gratings with the same period but different orientation. In Fig. 2(b) we show its optical Fourier transform obtained at the Fourier plane. We see the first harmonics of the scene forming a circle around the zero frequency. The second harmonics are also slightly visible. In Fig. 2(c) we show the resulting image when applying a filter which blocks the harmonics in some specific orientations. We clearly distinguish the gratings that are removed by this filtering operation. Finally, in Fig. 3 (setup in Fig. 1(b)) we show the transmitted image by a volume phase grating exhibiting maximum diffraction efficiency and oriented at the Bragg angle $\psi=\theta$ Bragg. We see that we obtain edge enhancement in the vertical edges.

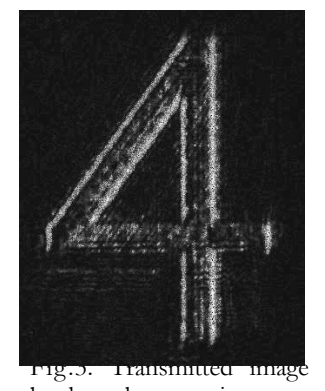

by the volume grating.

\section{Conclusions}

We have proposed the use of two different setups to show the students in optical image processing two complementary approaches to modify the frequency content of an object, with and without a Fourier plane, i.e. two different ways to synthesize the transfer function of a system. The understanding of the two experiments helps for a deeper understanding of optical image processing, its linear systems mathematical background, and some aspects of volume holography.

\section{Acknowledgments}

This work was supported by Generalitat Valenciana, Spain (projects GV01-130, GV04A/574 and GV04A/565).

\section{References}

J. W. Goodman, Introduction to Fourier Optics, McGraw-Hill, 2nd edition, New York, (1996). A. Márquez, J. Barbé, M.J. Yzuel and J. Campos, "Optical correlator as a tool for physicists and engineers training in signal processing", Proc. SPIE 3831, 297-306 (1999).

S. K. Case, "Fourier processing in the object plane", Opt. Lett. 4(9), 286-288 (1979).

H. Kogelnik, "Coupled wave theory for thick hologram gratings", Bell Syst. Technol. J. 48(9), 2909-2947 (1969).

A. Márquez, C. Neipp, S. Gallego, M. Ortuño, A. Beléndez and I. Pascual, "Edge enhanced imaging using PVA/acrylamide photopolymer gratings", Opt. Lett. 28(17), 1510-1512 (2003). 